\title{
Motorcortical Excitability and Synaptic Plasticity Is Enhanced in Professional Musicians
}

\author{
Karin Rosenkranz, ${ }^{1}$ Aaron Williamon, ${ }^{2}$ and John C. Rothwell ${ }^{1}$ \\ ${ }^{1}$ Sobell Department of Motor Neuroscience and Movement Disorders, Institute of Neurology, London WC1N 3B, United Kingdom, and ${ }^{2}$ Royal College of \\ Music, London SW7 2BS, United Kingdom
}

\begin{abstract}
Musicians not only have extraordinary motor and sensory skills, but they also have an increased ability to learn new tasks compared with non-musicians. We examined how these features are expressed in neurophysiological parameters of excitability and plasticity in the motor system by comparing the results of 11 professional musicians and 8 age-matched non-musicians. Parameters of motor excitability were assessed using transcranial magnetic stimulation (TMS) to measure motor-evoked potentials (MEPs) together with recruitment of corticospinal projections [input- output curve (IOcurve)] and of short-latency intracortical inhibition (SICIcurve). Plasticity, here defined as change of synaptic effectiveness, was tested by measuring MEPs and IOcurves after paired associative stimulation (PAS), which consists of an electric median nerve stimulus repeatedly paired (200 times at $0.25 \mathrm{~Hz}$ ) with a TMS pulse over the hand motor area. Using an interstimulus interval of $25 \mathrm{~ms}$ (PAS25) or $10 \mathrm{~ms}$ (PAS10), this leads to long-term potentiation- or long-term depression-like plasticity, respectively. Musicians showed steeper recruitment of MEPs and SICI (IOcurve and SICIcurve). Additionally, PAS25 increased and PAS10 decreased the MEP amplitudes and the slope of the IOcurves significantly more in musicians than in non-musicians. This is consistent with a wider modification range of synaptic plasticity in musicians. Together with the steeper recruitment of corticospinal excitatory and intracortical inhibitory projections, this suggests that they regulate plasticity and excitability with a higher gain than normal. Because some of these changes depend on age at which instrumental playing commenced and on practice intensity, they may reflect an increase in number and modifiability of synapses within the motor area caused by long-term musical practice.
\end{abstract}

Key words: motor cortex; excitability; plasticity; musician; transcranial magnetic stimulation; motor training

\section{Introduction}

Playing a musical instrument is one of the most complex skills a human can achieve and is the result of intense sensory and motor training often started at early age. Compared with nonmusicians, the musicians' brain shows structural and functional changes (Elbert et al., 1995; Schlaug et al., 1995; Jancke et al., 2000; Munte et al., 2002; Gaser and Schlaug, 2003; Haslinger et al., 2004; Ragert et al., 2004; Bengtsson et al., 2005; Bangert and Schlaug, 2006). For example, magnetic resonance (MR) imaging studies have highlighted an increase in gray matter volume in the sensorimotor cortex in musicians (Gaser and Schlaug, 2003) and an increased intrasulcal length of the precentral gyrus (Amunts et al., 1997); magneto-encephalographic studies have shown enlarged cortical somatosensory representations of fingers (Elbert et al., 1995). Not only do musicians have impressive motor skills and better somatosensory discrimination abilities than nonmusicians, they also show enhanced motor and sensory learning capabilities. Their tactile discrimination skills are improved pro-

\footnotetext{
Received 0ct. 18, 2006; revised March 31, 2007; accepted April 2, 2007.

This work was supported by Action Medical Research UK, the Dystonia Medical Research Foundation, the Bachmann-Strauss Dystonia and Parkinson Foundation, and the Medical Research Council.

Correspondence should be addressed to Dr. Karin Rosenkranz, Sobell Department of Motor Neuroscience and Movement Disorders, Institute of Neurology, 8-11 Queen Square, London WC1N 3B, UK. E-mail: k.rosenkranz@ion.ucl.ac.uk.

D0I:10.1523/JNEUROSCI.0836-07.2007

Copyright $\odot 2007$ Society for Neuroscience $\quad 0270-6474 / 07 / 275200-07 \$ 15.00 / 0$
}

portionally more by a tactile costimulation protocol than in nonmusicians (Ragert et al., 2004). Similarly, musicians who started musical training before 7 years of age learn a timed motor sequence task much better than musicians who started later or non-musicians (Watanabe et al., 2007).

In this study, we focused on the motor system and asked whether basic neurophysiological measures of motor cortex excitability and synaptic plasticity in musicians are changed in such a way as to contribute to their enhanced motor skills and learning abilities. We used transcranial magnetic stimulation (TMS) applied in single and paired-pulse paradigms to measure basic excitability parameters: input-output curves (IOcurves) test stimulus intensity-dependent recruitment of corticospinal projections to the small hand muscles (Ridding and Rothwell, 1997), whereas recruitment of short-latency intracortical inhibition (SICI) is measured in response to different conditioning stimulus intensities (SICIcurves) (Kujirai et al., 1993; Ziemann et al., 1996; Orth et al., 2003).

Motor cortical plasticity was assessed with the technique of paired associative stimulation (PAS), which consists of a median nerve stimulus followed by a single TMS pulse applied over the hand area of the contralateral motor cortex. Like associative synaptic conditioning in reduced animal preparations, the effects of PAS in humans depend on the interval between the sensory input and the magnetic pulse. Long-term potentiation (LTP)-like effects on cortical synapses occur with an interval of $25 \mathrm{~ms}$ between 
Table 1. Characteristics of musicians

\begin{tabular}{llllll}
\hline Musician & Male (M)/ & & Age when & Practice intensity \\
female (F) & Age (years) & Instrument & $\begin{array}{l}\text { started playing } \\
\text { over last year (hours/day) }\end{array}$ \\
\hline 1 & F & 25 & Piano & 6 & 3 \\
2 & M & 26 & Piano & 4 & 3 \\
3 & M & 24 & Piano & 5 & 4 \\
4 & F & 27 & Piano & 7 & 4 \\
5 & M & 19 & Piano & 5 & 4.5 \\
6 & F & 18 & Piano & 4 & 4 \\
7 & F & 23 & Piano/organ & 5 & 4.5 \\
8 & F & 24 & Recorder & 4 & 4.5 \\
9 & M & 34 & Trumpet & 10 & 0.5 \\
10 & F & 23 & Trombone & 11 & 4 \\
11 & F & 25 & Guitar & 12 & 5 \\
\hline
\end{tabular}

stimuli (PAS25), and long-term depression (LTD)-like effects emerge with an interval of $10 \mathrm{~ms}$ (PAS10) (Stefan et al., 2002; Wolters et al., 2003; Classen et al., 2004). Application of the PAS protocols interacts with short-term motor learning in humans (Ziemann et al., 2004; Stefan et al., 2006), and therefore the method seems likely to test circuits involved in natural behaviors.

We hypothesize that, as an expression of their structural and functional adaptation to musical training, musicians will show different recruitment of corticospinal and intracortical projections and, furthermore, that their synaptic plasticity will be higher than normal and could potentially contribute to their increased learning abilities in motor and sensory tasks.

\section{Materials and Methods}

Subjects. Eight healthy right-handed non-musicians (four females) aged 23-35 years and 11 musicians (seven females) aged 20-35 years gave informed consent for the study (see Table 1 for details on musicians' characteristics), which was approved by the joint ethics committee of the Institute of Neurology and National Hospital for Neurology and Neurosurgery, London. All experiments conform to the Declaration of Helsinki. The experiments were all performed in the morning, and none of the musicians had played their instrument directly beforehand. Subjects were comfortably seated in an armchair with their right forearm pronated and their hand relaxed on an armrest.

TMS. TMS was performed using two Magstim 200 stimulators (Magstim, Dyfed, UK) connected to a figure-eight-shaped coil with an internal wing diameter of $7 \mathrm{~cm}$ by a Y-cable. The coil was held with the handle pointing backward and laterally $\sim 45^{\circ}$ to the interhemispheric line to evoke anteriorly directed current in the brain and was optimally positioned to obtain motor-evoked potentials (MEPs) in the abductor pollicis brevis muscle (APB). Stimulation intensities are quoted in the text as a percentage of maximal stimulator output $( \pm S E)$.

Electromyographic recording. Surface electromyographic recordings in a belly-to-tendon montage were made from the APB, the first dorsal interosseus, and the abductor digiti minimi muscle of the right hand. The raw signal was amplified and filtered with a bandpass filter of $30 \mathrm{~Hz}$ to 1 $\mathrm{kHz}$ (Digitimer, Welwyn Garden City, UK). Signals were digitized at 2 $\mathrm{kHz}$ (CED Power 1401; Cambridge Electronic Design, Cambridge, UK) and stored on a laboratory computer for off-line analysis.

Experimental parameters. The MEPs and IOcurves were measured before and after the PAS protocol (see below). At the beginning of each experiment, the stimulus intensity that evokes an MEP of $\sim 1 \mathrm{mV}$ peakto-peak amplitude (SI1mV) was defined. Using SI1mV, 15 MEPs were recorded before and after each PAS protocol, and the mean amplitude was calculated for the data obtained before and after PAS in each single subject. For the IOcurves, the intensities of the single TMS stimuli were individually adapted according to the predefined SI $1 \mathrm{mV}$. Ten MEPs each were recorded with $50,70,80,90,100(=S I 1 \mathrm{mV}), 110,120,130$, and $150 \%$ of SI $1 \mathrm{mV}$. For each subject, the peak-to-peak amplitudes were measured on each single trial to calculate the mean amplitude for each stimulation intensity.
The SICIcurve was measured on the first experimental session before the PAS protocol. A subthreshold conditioning stimulus preceded the suprathreshold test stimulus for $3 \mathrm{~ms}(\mathrm{Ku}-$ jirai et al., 1993). The test stimulus intensity was always set at SI $1 \mathrm{mV}$, but the intensity of the conditioning stimulus was varied. Ten trials each were recorded in blocks with 70, 80, and $90 \%$ of the active motor threshold (aMT). The aMT was defined as the minimum intensity needed to evoke an MEP of $>200 \mu \mathrm{V}$ in 5 of 10 trials in the tonically active APB $(\sim 20 \%$ of maximal contraction as assessed visually on an oscilloscope). Before, between, and after the blocks, five single test pulses were given to ensure that the unconditioned MEP size was stable. The peak-to-peak amplitude of the conditioned and test MEPs was measured for each single trial to calculate the mean amplitude and percentage SICI for the three different conditioning stimulus intensities.

PAS. PAS consisted of 200 electrical stimuli of the right median nerve at the wrist paired with a single TMS pulse over the hot spot of the APB area of the left hemisphere with a rate of $0.25 \mathrm{~Hz}$. Electrical stimulation was applied through a bipolar electrode (cathode proximal), using square-wave pulses (duration, $0.2 \mathrm{~ms}$ ) at an intensity of three times the perceptual threshold. TMS was delivered through a figure-eight coil (diameter of each wing, $70 \mathrm{~mm}$ ) connected to a Magstim 200 magnetic stimulator (Magstim) and was held in the same position as described above. Stimulation was applied at an intensity adjusted to evoke an MEP of SI $1 \mathrm{mV}$ in the relaxed APB. Subjects took part in two experimental sessions that were separated by at least 1 week. In randomized order, the effect of PAS given with an interstimulus interval of $25 \mathrm{~ms}$ (PAS25) and of $10 \mathrm{~ms}$ (PAS10) between peripheral and TMS stimulus was tested. The former has been shown previously to induce long-lasting MEP increase (Stefan et al., 2000, 2002), and the latter has previously shown an MEP decrease (Wolters et al., 2003). Subjects were instructed to look at their stimulated hand and count the peripheral electrical stimuli they perceived. The MEPs evoked in the APB were displayed on-line during the intervention to control for the correct coil position and stored for off-line analysis.

Data analysis and statistics. The comparability of the subject groups for age was tested by an unpaired $t$ test. The aMT and intensities of conditioning and test stimuli (SI $1 \mathrm{mV})$ are given as the percentage of stimulator output. Comparability between the groups, as well as within the groups for each experimental part (PAS25 and PAS10), was tested by means of unpaired and paired two-tailed $t$ tests, respectively. For statistical analysis, repeated-measures ANOVA, with the between-subject factor "group," were performed as two-way ANOVAs. Where appropriate, additional one-way ANOVAs and post hoc $t$ tests were applied, as outlined in detail in Results.

To simplify the data set obtained by measuring the IOcurves and the SICI curves, the slopes of these curves measured as the steepness of the linear regression line through the given data points [for input-output slope (IOslope) between 90 and 130\% SI $1 \mathrm{mV}$; for the SICIcurves for all three SICI levels measured with 70, 80, and 90\% aMT] were calculated.

For the data obtained in musicians, correlation analyses were performed on performance (starting age, practice intensity) and experimental parameters, and Pearson's correlation coefficient $(r)$ was calculated (see Results for details). We set the significance levels for the ANOVAs to $p<0.01$ to correct for multiple comparisons and for the $t$ tests to $p<$ 0.05 . All data are given as means \pm SE.

\section{Results}

None of the subjects experienced any side effects from TMS during the experiments. There was no difference between the groups in age (non-musicians, $27.6 \pm 2.2$ years; musicians, $24.7 \pm 1.2$ years), aMT (non-musicians, $38.8 \pm 2.7 \%$ stimulator output; musicians, $36.1 \pm 1.8 \%$ stimulator output), or intensity of the 


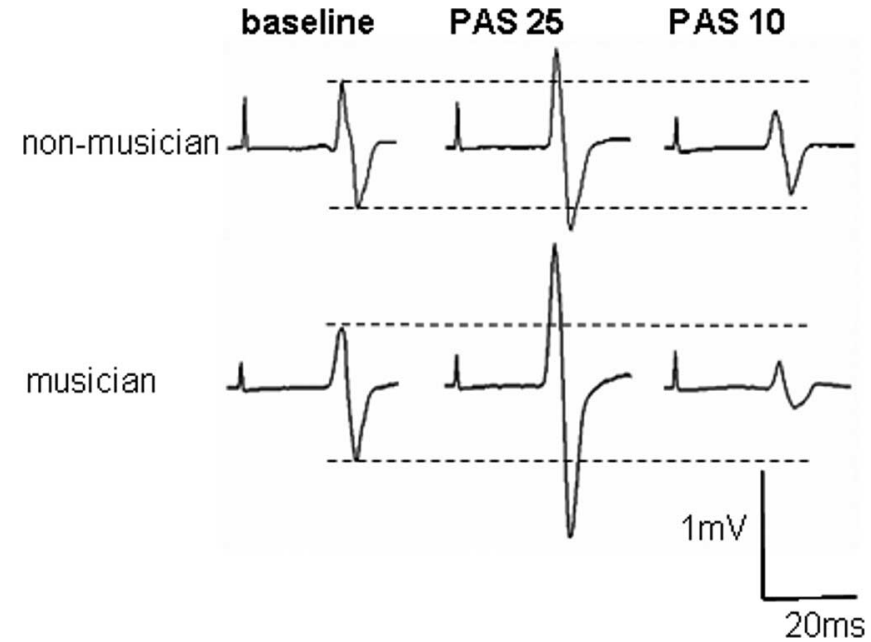

Figure 1. Average MEP recordings from the APB of one representative non-musician and one musician. The TMS intensity was adjusted to evoke a baseline MEP with peak-to-peak amplitude of $1 \mathrm{mV}$ in the APB as the main target muscle. After PAS25, the MEP amplitudes were enhanced, and after PAS10, they were reduced in both subjects. However, both effects were much larger in the musician than the non-musician.
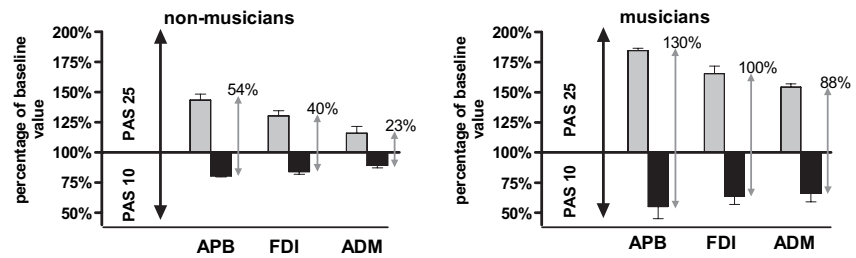

Figure 2. Mean MEP ( $\pm S E$ ) in non-musicians and musicians for all three intrinsic hand muscles. Changes after PAS25 (gray) and PAS10 (black) are expressed as a percentage increase/ decrease in the baseline MEP. The double-headed arrows show the mean total range of modulation by the PAS interventions. In both groups of subjects, PAS produced the largest effects on MEPs in the median nerve-innervated APB than the other two muscles. But comparing both groups, the effects were greater in musicians than in non-musicians.

test stimulus needed to evoke an MEP of $\sim 1 \mathrm{mV}$ peak-to-peak amplitude in the APB (non-musicians, $54.3 \pm 4.4 \%$ stimulator output; musicians, $54.7 \pm 3.5 \%$ stimulator output). The number of peripheral nerve stimuli counted during the PAS protocols was not different in non-musicians (PAS25, $200 \pm 1$; PAS10, $201 \pm 1$ ) and musicians (PAS25, $200 \pm 1$; PAS10, $199 \pm 1$ ).

\section{Effect of PAS25 and PAS10 on MEPs}

Figure 1 illustrates the main finding with original recordings from one representative non-musician and one musician before and after PAS25 and PAS10. In both subjects, MEPs recorded in the APB are facilitated after PAS25 and suppressed after PAS10. However, the effect is much larger in the musician than the non-musician.

Figure 2 shows the mean ( $\pm \mathrm{SE}$ ) data in both groups of subjects for all three intrinsic hand muscles. In each muscle, facilitation of MEPs after PAS25 is expressed as a percentage increase in the baseline MEP; suppression after PAS10 is expressed as percentage reduction in MEP.

In each subject, baseline MEPs were of similar size before PAS25 and PAS10 ( $t$ tests, $p>0.46$ ); this allowed this simplified display of the PAS25 and PAS10 effect as double-headed arrows, indicating the total range of modulation by the PAS interventions (PAS range).

In both groups of subjects, PAS produced a larger effect on MEPs in the median nerve-innervated APB than the other two muscles, but in all cases, the effects were greater in musicians than in nonmusicians, which is most clearly seen by comparing the PAS range.

This was confirmed in the two-way ANOVA on the PAS range, with the factors group and "muscle." There was a significant main effect of both group $\left(F_{(1,4)}=3.41 ; p=0.0007\right)$ and muscle $\left(F_{(2,8)}=25.60 ; p=0.0003\right)$ but no significant interaction, indicating that the effect of PAS was distributed similarly among the recorded hand muscles in the two groups, although it was stronger in the musicians. A subsequent one-way ANOVA for each group separately showed a significant effect of muscle in both groups (non-musicians: $F_{(2,10)}=18.89, p=0.004$; musicians: $\left.F_{(2,16)}=15.71, p=0.0002\right)$, showing that the distribution of the PAS effect, which was strongest in the APB and weakest in the $\mathrm{ADM}$, is significant in both groups. Comparing musicians and non-musicians, PAS25-induced facilitation, PAS10-induced inhibition, and PAS range were in all recorded muscles significantly higher in musicians ( $t$ tests, $p<0.001$ ).

\section{Effects of PAS25 and PAS10 on IOcurves}

Figure 3, $A$ and $B$, shows the effect of PAS25 and PAS10 on the IOcurves of the APB in both groups of subjects. The slopes of the curves are increased after PAS25 and decreased after PAS10.

Interestingly the slopes of the baseline IOcurves appear to be steeper in musicians. To analyze these data, we first noted that in each group the baseline IOcurves were very similar before PAS25 and PAS10. This was confirmed by a within-group two-way ANOVA with the factors "stimulus intensity" and "protocol." There was a significant main effect of stimulus intensity $\left(F_{(1,8)}=\right.$ 72.19; $p<0.0001$ ), but no effect of protocol, nor a significant interaction. This allowed us to pool the two sets of baseline data in each group into a single baseline IOcurve as shown in Figure 3C. The slope of the IOcurve for musicians was steeper than that for the non-musicians. Thus, two-way ANOVA with the factors group and stimulus intensity showed a significant main effect for both factors (group: $F_{(1,15)}=20.61, p<0.0001$; stimulus intensity: $\left.F_{(1,8)}=107.23, p<0.0001\right)$, as well as a significant interaction $\left(F_{(8,120)}=3.82 ; p=0.0004\right)$. Post hoc tests at each intensity revealed that MEPs were larger in musicians at stimulus intensities of 120 and $130 \%$ aMT ( $t$ test, $p<0.025$ ). Given that the slope of the IOcurve was approximately linear between 90 and $130 \%$ aMT (Fig. $3 A-C$, gray box), we conducted the remaining analysis on the slopes of the curves in each condition.

Figure $3 D$ shows the mean slope of the IOcurves before and after PAS25 or PAS10 for non-musicians and musicians. As noted above using the ANOVA analysis, the slope of the baseline IOcurve in musicians was significantly steeper than in nonmusicians ( $t$ test, $p<0.01$ ). After PAS25, the slope increased in both groups, although this was only significant in musicians $(t$ test, $p=0.0001)$; PAS10 decreased the slope significantly in both groups ( $t$ tests; non-musicians, $p=0.0125$; musicians, $p=$ 0.0002 ). Figure $3 E$ shows the percentage change in slope relative to baseline after PAS. In musicians, PAS changed the slope more than in non-musicians. A two-way ANOVA with the factors group and protocol showed no significant main effect of group $\left(F_{(1,5)}=0.19 ; p=0.678\right)$ but a significant main effect of protocol $\left(F_{(1,5)}=80.46 ; p=0.0003\right)$ and a significant group $\times$ protocol interaction $\left(F_{(1,5)}=31.62 ; p=0.0025\right)$. This was because the PAS25 protocol produced a stronger increase ( $t$ test, $p=0.03$ ) and the PAS10 protocol produced a stronger decrease in slope of the IOcurve in musicians compared with non-musicians ( $t$ test, $p=0.01)$. 

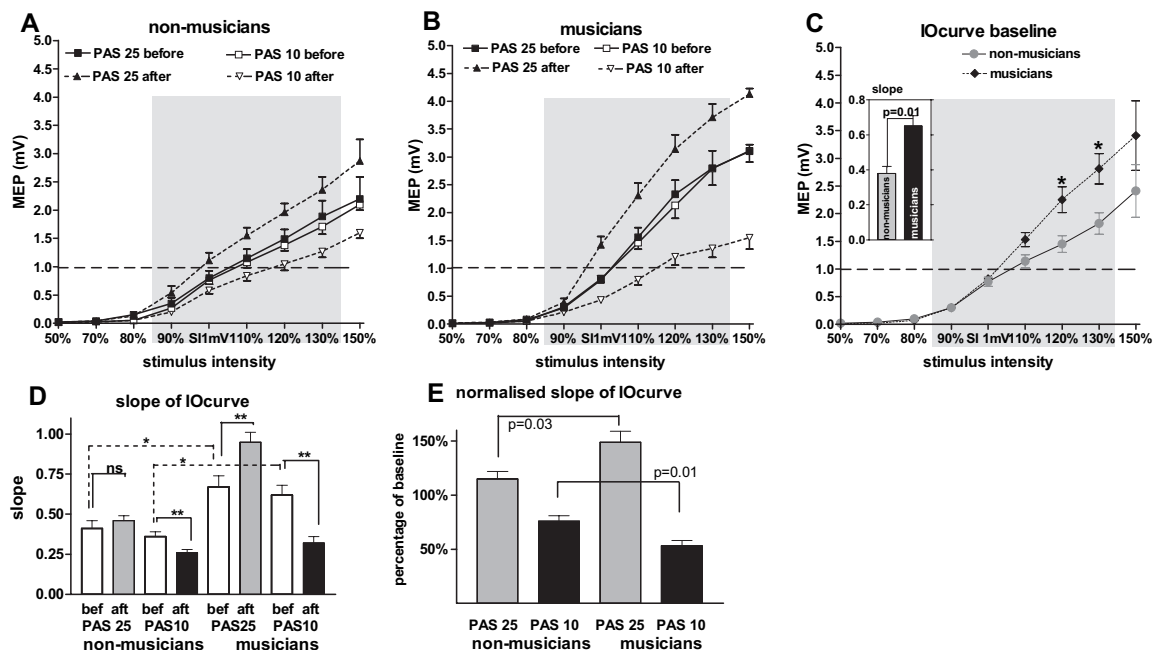

Figure 3. IOcurves in both groups before and after PAS. $A-C$, The mean MEP amplitude ( $\pm S E)$ as given on the $y$-axis against the stimulus intensity given on the $x$-axis (in percentage of $S 11 \mathrm{mV}$ ). $A, B, 10$ curves measured before and after PAS25 and PAS10, respectively, for non-musicians $(\boldsymbol{A})$ and for musicians $(\boldsymbol{B})$. Musicians showed a steeper increase in both of the 10 curves measured before PAS, as well as after PAS25. In both groups, the IOcurves measured before the PAS were not significantly different. $C$, Pooled IOcurves before PAS for each group separately. The slope of the curve has been calculated for the approximately linear part between 90 and 130\% SI1mV and is shown in the inserted column graph for non-musicians (gray) and musicians (black). The mean MEP amplitudes were significantly higher in musicians at stimulus intensities of $110-130 \% \mathrm{SI} 1 \mathrm{mV}\left(t\right.$ test, $\left.{ }^{*} p \leq 0.05\right)$, as was the slope of the pooled IOcurve ( $t$ test, $p=0.01$ ). $\boldsymbol{D}$, The slopes for all IOcurves as shown in $\boldsymbol{A}$ and $\boldsymbol{B}$ for non-musicians and musicians. Statistical results of direct comparisons of the slopes before and after PAS within the groups as well as of direct comparisons between the groups are shown $\left(t\right.$ test; $\left.{ }^{*} p \leq 0.05 ; * * 0.01\right)$. $\boldsymbol{E}$, The slopes after PAS as a percentage of the slope measured before. Compared with non-musicians, the musicians showed a significantly stronger slope increase after PAS25 and decrease after PAS10 ( $t$ test; $p$ values shown).

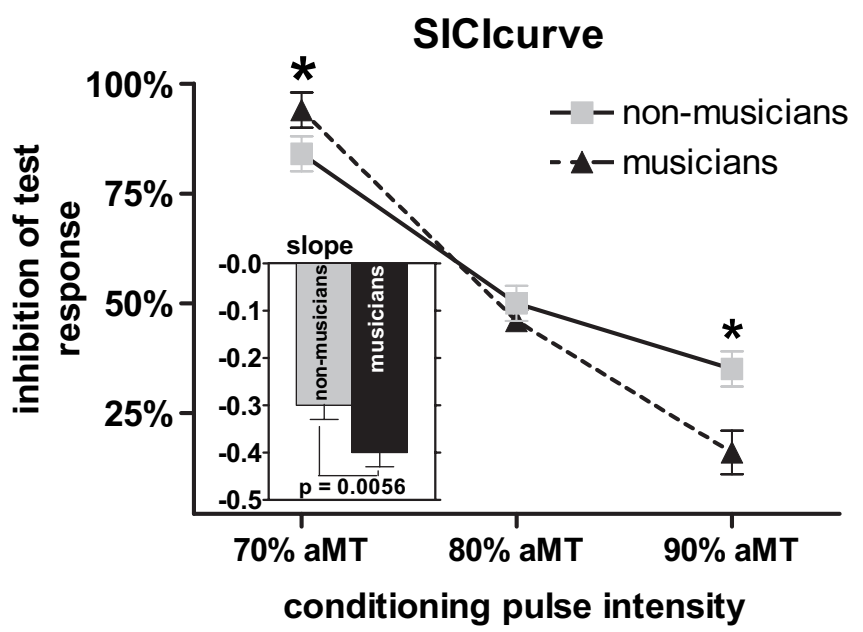

Figure 4. The SICl obtained with a conditioning pulse intensity of 70,80 , and $90 \%$ aMT is shown as the percentage of MEP evoked by the test pulse alone ( \pm SE). With a conditioning stimulus of 70\% aMT, musicians showed less, with $90 \%$ aMT more SICl than non-musicians, whereas at $80 \%$ aMT the level of SICI was similar in both groups ( $t$ test, $\left.{ }^{*} p \leq 0.015\right)$. The slope calculated for the SIClcurve described when displaying the amount of $\mathrm{SICl}$ against the conditioning stimulus intensity was significantly steeper in musicians (inserted column graph; $t$ test, $p=0.0056)$.

\section{SICIcurves}

The amplitude of the MEP evoked in APB by the test pulse alone $(\mathrm{SI} 1 \mathrm{mV})$ was the same in non-musicians and musicians. This allows to display the SICI data obtained with a conditioning stimulus intensity of 70,80 , and $90 \%$ aMT as percentage of control values obtained with the test stimulus alone (Fig. 4). Increasing the intensity of the conditioning stimulus increases the amount of inhibition in both groups, but the slope of the relationship is steeper in musicians. This was confirmed by a two-way ANOVA with the factors group and "conditioning pulse intensity". There was a significant main effect of conditioning pulse intensity $\left(F_{(2,10)}=821.18 ; p<\right.$ $0.0001)$, as well as a significant interaction $\left(\right.$ ANOVA; $\left.F_{(2,10)}=4.76 ; p=0.035\right)$. The fact that there was no significant main effect of group is likely to be attributable to the fact that musicians had significantly less inhibition at $70 \%$ aMT ( $t$ test, $p=0.004$ ) and significantly more inhibition at $90 \%$ aMT $(t$ test, $p=0.015$ ) with no difference between groups at $80 \%$ aMT ( $t$ test, $p=0.14$ ). We also calculated the slope of the SICIcurve and found that it was steeper in musicians than in non-musicians ( $t$ test, $p=0.0056$ ).

\section{Correlations with performance parameters in musicians}

Figure $5, A$ and $B$, shows the correlation between the age at which instrumental playing was commenced with the experimental parameters SICIslope and IOslope before and after PAS. There were significant correlations between starting age and the slope of the SICIcurve $(r=0.86 ; p=$ 0.0008 ) and between starting age and the normalized slope of the IOcurve after $\operatorname{PAS} 25(r=-0.63 ; p=0.036)$ : the earlier playing was started, the higher was the increase in slope of the IOcurve after PAS25 and the steeper was the slope of the SICIcurve.

However, the seven pianists (Fig. $5 A, B$, filled symbols) started playing earlier than the other four instrumentalists (Fig. $5 A, B$, open symbols); thus, an instrument-specific effect cannot be completely ruled out, although the correlation between the starting age and the IOslope change after PAS was significant for non-pianists alone $(r=-0.97 ; p=0.034)$.

Figure $5 C$ shows the correlation between the actual amount of playing within the last 5 years with the PAS range: the more intensive the musician played, the wider was the range of MEP changes induced by the PAS protocols $(r=0.62 ; p=0.034)$. This correlation was independent of the instrument and was significant for pianists $(r=0.95 ; p=0.0008)$ and non-pianists $(r=$ $0.73 ; p=0.041)$.

\section{Discussion}

There were two main findings in the present data. First, the IOcurve and the SICIcurve were steeper in musicians compared with non-musicians. Second, PAS25- and PAS10-induced effects on MEP amplitudes and slope of the IOcurve were stronger in musicians. These features are compatible with the idea that both motorcortical excitability and plasticity are heightened in musicians. Because some of these changes depend on the age at which instrumental playing commenced, we hypothesize that they reflect adaptation to long-term musical practice.

\section{Differences of baseline excitability in non-musicians and musicians}

The slope of the IOcurve depends on the distribution of excitability in populations of motoneurons and interneurons at spinal and cortical sites and is therefore influenced by many factors 
(Ridding and Rothwell, 1997; Boroojerdi et al., 2001; Jensen et al., 2005). There are no detailed studies of spinal excitability in musicians, so it remains possible that their steeper IOcurves are attributable to changes in the excitability of spinal motoneurons such that a larger response is evoked by a given corticospinal volley. Although subthreshold differences in the distribution of excitability in the spinal motoneuron pool between musicians and non-musicians cannot be excluded, we tried to control for this by expressing stimulus intensities relative to the SI $1 \mathrm{mV}$ in APB, which was similar in both groups. Therefore, it seems likely that some of the increased slope of the IOcurve in musicians relates to changes in motor cortical organization.

Effects on the cortex are consistent with animal studies showing that the late phase of motor skill learning is associated with increases in the number of synapses in the corresponding area of the motor cortex (Kleim et al., 2002, 2004). The increased gray matter density found in MR images of musicians' brains suggests that a similar process occurs in them. Indeed, changes in gray matter are larger in musicians who started earlier (Gaser and Schlaug, 2003), and they reflect the choice of instrument (Bangert and Schlaug, 2006), consistent with a causal connection between presumed synaptic growth and the duration and pattern of training. If so, then it may be that increased synaptic connectivity is one factor that leads to increased recruitment in musicians.

SICI tests the excitability of local GABA $\mathrm{A}_{\mathrm{A}}$ ergic inhibitory circuits in the motor cortex (Hanajima et al., 1998; Ilic et al., 2002). The amount of SICI that is evoked depends on the stimulus intensity of the conditioning pulse, describing a "U-shaped" curve (Kujirai et al., 1993; Ziemann et al., 1996; Ilic et al., 2002; Orth et al., 2003) of which the present experiments explored the initial increase in SICI toward its peak level. Recruitment of SICI represents a tradeoff between recruitment of inhibitory and excitatory elements as the conditioning intensity rises (Ilic et al., 2002). In our study, we expressed the intensity of the conditioning stimulus relative to aMT, which had the same absolute value in musicians and non-musicians. This yielded an SICIcurve that was steeper in musicians: lower conditioning pulse intensities evoked less SICI than in non-musicians, whereas higher intensities evoked more SICI. This difference would not have been noted if we had compared the groups at only median conditioning stimulus intensity, thus reinforcing the importance of measuring the SICI with different conditioning pulse intensities.

The difference between groups may reflect differences in the threshold and/or distribution of excitability in populations of cortical interneurons. If so, we favor the possibility that, like the IOcurves, increasing the conditioning stimulus intensity recruits more intracortical inhibitory connections because of increased synpatic density in the cortex. However, the finding that at lowest conditioning stimulus intensity the SICI in musicians is not as strong as it is in non-musicians shows that the threshold above which these intracortical inhibitory projections get activated is higher in musicians.

In summary, the combination of an increased slope for both the corticospinal (IOcurve) and intracortical (SICIcurve) inputoutput relationships suggests that regulation of cortical output is
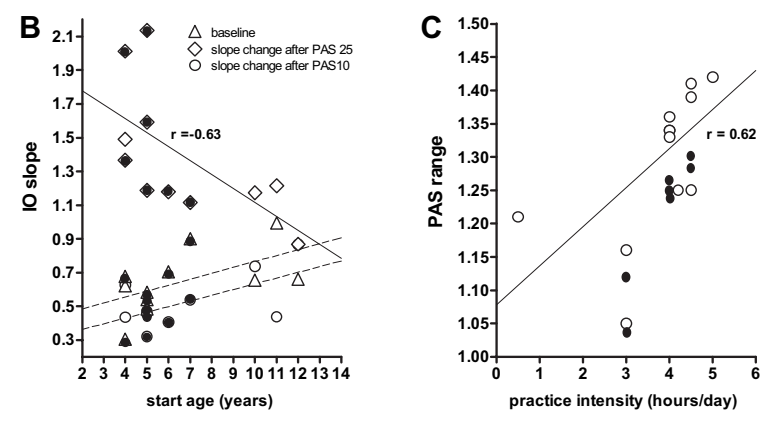

Figure 5. Correlation between musicians' performance parameter and measures of motor excitability and plasticity. Filled symbols represent pianists, and open symbols represent other instrumentalists (two brass players, one guitarist, one recorder before and after PAS. Musicians who started at a younger age had a steeper SICIslope and also a stronger IOslope ( practice more intensively had a significantly higher MEP range, an effect that was unrelated to the instrument played. Pearson's are given for significant correlations.

more sensitive, or has a higher gain, in musicians than in nonmusicians: that is, small changes in TMS intensity, either as a single or conditioning pulse, have a proportionally stronger effect on cortical output in musicians. This may favor rapid recruitment of corticospinal output during recruitment of the motor cortex in volitional movement. The fact that SICI is recruited at higher thresholds, but with increased gain, might complement this quick recruitment with a powerful brake to prevent unwanted spread of activation, consistent with the fact that musicians show more focused motorcortical activation during movement (Jancke et al., 2000; Krings et al., 2000; Lotze et al., 2003; Meister et al., 2005)

\section{Differences in the effect of PAS in musicians and non-musicians}

To our knowledge, the PAS effect in humans has only been measured as amplitude change of MEPs evoked by a standard test pulse (Stefan et al., 2000, 2002, 2006; Wolters et al., 2003; Ziemann et al., 2004). However, the interpretation of any differences in PAS between groups of individuals depends on whether the slopes of the IOcurves are equal in the groups. For example, using the known effect of increasing TMS intensity on the MEP size as a model for the PAS effect, then as illustrated in Figure 6, an increase in the test pulse by $20 \%$ would increase MEP by $50 \%$ in groups with "standard" IOslope. However, if the IOslope were twice as steep, then the increase in MEP would be $100 \%$.

Because in musicians and non-musicians the slopes of the baseline IOcurve were different, it was important to test the effect of PAS over the entire range of input intensities. We found that in musicians, PAS increased the slope of the IOcurve significantly more and therefore that PAS had a greater effect than in nonmusicians. If the steeper baseline IOcurve in musicians reflects an increased number of interneuronal connections caused by adaptation to long-term musical training, a proportionally stronger PAS effect suggests that these synapses also have a higher propensity to undergo changes in synaptic efficacy.

The neural circuits involved in PAS and short-term behavioral motor learning overlap. Previous short-term motor learning occludes or reverses the ability of PAS to induce LTP-like plasticity, whereas it may have no effect or even increase LTD-like plasticity (Ziemann et al., 2004; Stefan et al., 2006). The musicians tested here started their musical training at an average age of $\sim 6.5$ years, and by the age of 20 years, Ericsson et al. (1993) estimated they 


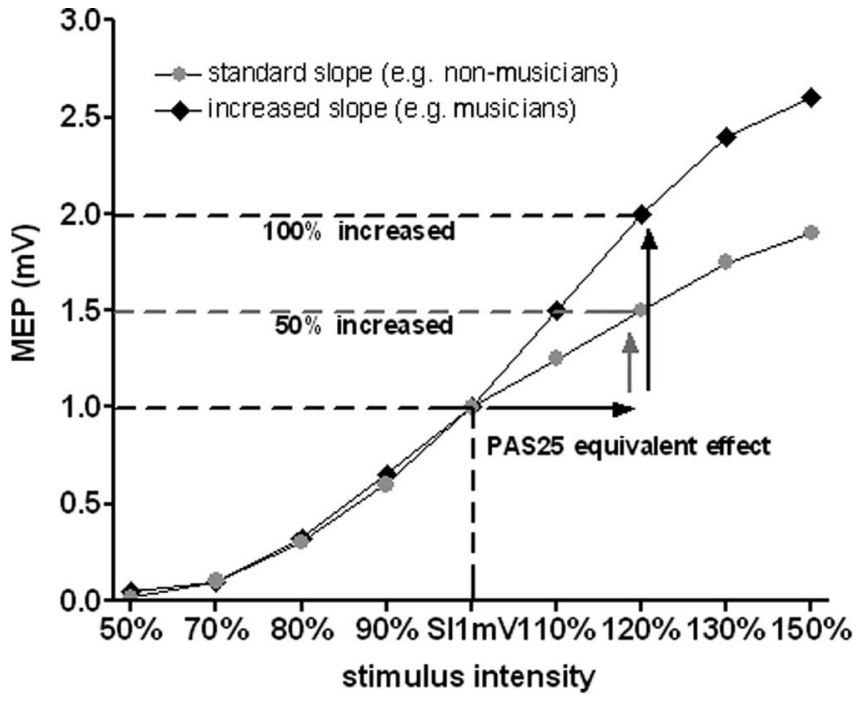

Figure 6. Effect of the baseline slope of the I0curve on the MEP effects of PAS25. The diagram shows hypothetical I0curves of the two different groups of subjects (gray and black) that differ in slope. In this example, PAS25 causes an equivalent 20\% increase in the effectiveness of a TMS stimulus. This leads to a 50\% increase in MEP amplitude in the gray group but to a $100 \%$ increase in the black group. Thus, if the PAS25 effect was only measured by its effect on MEPs elicited at a single intensity of test stimulus (e.g., a preinterventional SI1mV), the result would suggest that PAS25 is more powerful in the black group than in the gray group. However, the difference may simply reflect a difference in initial I0curves rather than a difference in synaptic plasticity. A more complete demonstration of increased synaptic plasticity involves showing that PAS increases the slope of the I0curve (see text).

will have had an average of 10,000 h of practice. If occlusion between periods of learning were to occur as in non-musicians, then one would have to conclude that many of these hours of practice failed to achieve any effective outcome. However, because the number of hours of practice is well known to relate to musical skill, this seems unlikely. It may be that increased susceptibility for synaptic potentiation/depotentiation in musicians represents an adaptation to these learning demands and prevents occlusion from occurring. This might enable them to adapt motor performance quickly while preserving a high level of proficiency (e.g., when playing on an unfamiliar instrument).

\section{Nature versus nuture?}

Our experiments do not directly assess whether increased synaptic plasticity is produced by musical training, as a complex motor task and also a multimodal-sensory and emotional experience, or whether it is a genetic trait of successful musicians. However, some parameters correlated significantly with the age at which playing commenced as well as with practice intensity over the last 5 years.

Similarly, structural and functional changes in the brain of musicians are linked to performance parameters (Elbert et al., 1995; Gaser and Schlaug, 2003; Bangert and Schlaug, 2006). Although this does not completely rule out any preselectional bias, it seems likely that some of the changes of excitability and plasticity are a consequence of long-term musical practice.

\section{Beneficial versus maladaptive?}

Increased LTP-/LTD-like effects of PAS have been described in focal hand dystonia patients, where it is proposed that they may contribute to development of symptoms (Quartarone et al., 2006; Weise et al., 2006) and has even been interpreted as endophenotypic trait for focal dystonia (Quartarone et al., 2006).
However, the present data show increased plasticity in highly skilled musicians, implying that it can be associated with either maladaptive or beneficial changes. If so, then one tentative conclusion must be that other factors determine which predominates. One factor that may influence the behavioral effectiveness of enhanced PAS is the corresponding gain of inhibitory mechanisms. The steep slope of the SICIcurve in musicians may allow more effective control of enhanced plasticity and benefit performance. This might be not the case in focal dystonia, because some studies have described reduced SICI at rest (Ridding et al., 1995; Gilio et al., 2003), although this finding is inconsistent (Stinear and Byblow, 2004a,b; Butefisch et al., 2005; Rosenkranz et al., 2005).

Alternatively, aberrant plasticity might not have an early and primary pathogenic role in focal dystonia but evolve secondarily, a consequence rather than a cause of the disease (Weise et al., 2006).

\section{Conclusion}

In musicians, motorcortical excitability operates with a higher gain than normal. Furthermore, they show a higher sensitivity toward induction of plasticity using the PAS protocol. These changes may represent a beneficial adaptation in response to long-term musical training and support their excellent movement skills.

\section{References}

Amunts K, Schlaug G, Jancke L, Stein H (1997) Motor cortex and hand motor skills: structural compliance in the human brain. Hum Brain Mapp 5:206-215.

Bangert M, Schlaug G (2006) Specialization of the specialized in features of external human brain morphology. Eur J Neurosci 24:1832-1834.

Bengtsson SL, Nagy Z, Skare S, Forsman L, Forssberg H, Ullen F (2005) Extensive piano practicing has regionally specific effects on white matter development. Nat Neurosci 8:1148-1150.

Boroojerdi B, Ziemann U, Chen R, Butefisch CM, Cohen LG (2001) Mechanisms underlying human motor system plasticity. Muscle Nerve 24:602-613.

Butefisch CM, Boroojerdi B, Chen R, Battaglia F, Hallett M (2005) Taskdependent intracortical inhibition is impaired in focal hand dystonia. Mov Dis 20:545-551.

Classen J, Wolters A, Stefan K, Wycislo M, Sandbrink F, Schmidt A, Kunesch E (2004) Paired associative stimulation. Suppl Clin Neurophysiol 57:563-569.

Elbert T, Pantev C, Wienbruch C, Rockstroh B, Taub E (1995) Increased cortical representation of the fingers of the left hand in string players. Science 270:305-307.

Ericsson KA, Krampe RT, Tesch-Römer C (1993) The role of deliberate practice in the acquisition of expert performance. Psychol Rev 100:363-406

Gaser C, Schlaug G (2003) Brain structures differ between musicians and non-musicians. J Neurosci 23:9240-9245.

Gilio F, Rizzo V, Siebner HR, Rothwell JC (2003) Effects on the right motor hand-area excitability produced by low-frequency rTMS over human contralateral homologous cortex. J Physiol (Lond) 551:563-573.

Hanajima R, Ugawa Y, Terao Y, Sakai K, Furubayashi T, Machii K, Kanazawa I (1998) Paired-pulse magnetic stimulation of the human motor cortex: differences among I waves. J Physiol (Lond) 509:607-618.

Haslinger B, Erhard P, Altenmueller E, Hennenlotter A, Schwaiger M, Graefin von Einsiedel H, Rummeny E, Conrad B, Ceballos-Baumann AO (2004) Reduced recruitment of motor associtation areas during bimanual coordination in concert pianists. Hum Brain Mapp 22:206-215.

Ilic TV, Meintzschel F, Cleff U, Ruge D, Kessler KR, Ziemann U (2002) Short-interval paired-pulse inhibition and facilitation of human motor cortex: the dimension of stimulus intensity. J Physiol (Lond) 545:153-167.

Jancke L, Shah NJ, Peters M (2000) Cortical activations in primary and secondary motor areas for complex bimanual movements in professional pianists. Brain Res Cogn Brain Res 10:177-183. 
Jensen JL, Marstrand PCD, Nielsen JB (2005) Motor skill training and strength training are associated with different plastic changes in the central nervous system. J Appl Physiol 99:1558-1568.

Kleim JA, Barbay S, Cooper NR, Hogg TM, Reidel CN, Remple MS, Nudo RJ (2002) Motor learning-dependent synaptogenesis is localized to functionally reorganized motor cortex. Neurobiol Learn Mem 77:63-77.

Kleim JA, Hogg TM, Vandenberg PM, Cooper NR, Bruneau R, Remple M (2004) Cortical synaptogenesis and motor map reorganization occur during late, but not early, phase of motor skill learning. J Neurosci 24:628-633.

Krings T, Topper R, Foltys H, Erberich S, Sparing R, Willmes K, Thron A (2000) Cortical activation patterns during complex motor tasks in piano players and control subjects. A functional magnetic resonance imaging study. Neurosci Lett 278:189-193.

Kujirai T, Caramia MD, Rothwell JC, Day BL, Thompson PD, Ferbert A, Wroe S, Asselman P, Marsden CD (1993) Corticocortical inhibition in human motor cortex. J Physiol (Lond) 471:501-519.

Lotze M, Scheler G, Tan HR, Braun C, Birbaumer N (2003) The musician's brain: functional imaging of amateurs and professionals during performance and imagery. NeuroImage 20:1817-1829.

Meister I, Krings T, Foltys H, Boroojerdi B, Muller M, Topper R, Thron A (2005) Effects of long-term practice and task complexity in musicians and nonmusicians performing simple and complex motor tasks: implications for cortical motor organization. Hum Brain Mapp 25:345-352.

Munte TF, Altenmuller E, Jancke L (2002) The musician's brain as a model of neuroplasticity. Nat Rev Neurosci 3:473-478.

Orth M, Snijders AH, Rothwell JC (2003) The variability of intracortical inhibition and facilitation. Clin Neurophysiol 114:2362-2369.

Quartarone A, Siebner HR, Rothwell JC (2006) Task-specific hand dystonia: can too much plasticity be bad for you? Trends Neurosci 29:192-199.

Ragert P, Schmidt A, Altenmuller E, Dinse HR (2004) Superior tactile performance and learning in professional pianists: evidence for metaplasticity in musicians. Eur J Neurosci 19:473-478.

Ridding MC, Rothwell JC (1997) Stimulus/response curves as a method of measuring motor cortical excitability in man. Electroencephalogr Clin Neurophysiol 105:340-344.

Ridding MC, Sheean G, Rothwell JC, Inzelberg R, Kujirai T (1995) Changes in the balance between motor cortical excitation and inhibition in focal, task specific dystonia. J Neurol Neurosurg Psychiatry 59:493-498.

Rosenkranz K, Williamon A, Butler K, Cordivari C, Andrew JL, Rothwell JC (2005) Pathophysiological differences between musician's dystonia and writer's cramp. Brain 128:918-931.

Schlaug G, Jancke L, Huang Y, Staiger JF, Steinmetz H (1995) Increased corpus callosum size in musicians. Neuropsychologia 33:1047-1055.

Stefan K, Kunesch E, Cohen LG, Benecke R, Classen J (2000) Induction of plasticity in the human motor cortex by paired associative stimulation. Brain 123:572-584.

Stefan K, Kunesch E, Benecke R, Cohen LG, Classen J (2002) Mechanisms of enhancement of human motor cortex excitability induced by interventional paired associative stimulation. J Physiol (Lond) 543:699-708.

Stefan K, Wycislo M, Gentner R, Schramm A, Naumann M, Reiners K, Classen J (2006) Temporary occlusion of associative motor cortical plasticity by prior dynamic motor training. Cereb Cortex 16:376-385.

Stinear CM, Byblow WD (2004a) Impaired modulation of intracortical inhibition in focal hand dystonia. Cereb Cortex 14:555-561.

Stinear CM, Byblow WD (2004b) Elevated threshold for intracortical inhibition in focal hand dystonia. Mov Disord 19:1312-1317.

Watanabe D, Savion-Lemieux T, Penhune VB (2007) The effect of early musical training on adult motor performance: evidence for a sensitive period in motor learning. Exp Brain Res 176:332-340.

Weise D, Schramm A, Stefan K, Wolters A, Reiners K, Naumann M, Classen J (2006) The two sides of associative plasticity in writer's cramp. Brain 129:2709-2721.

Wolters A, Sandbrink F, Schlottmann A, Kunesch E, Stefan K, Cohen LG, Benecke R, Classen J (2003) A temporally asymmetric Hebbian rule governing plasticity in the human motor cortex. J Neurophysiol 89:2339-2345.

Ziemann U, Rothwell JC, Ridding MC (1996) Interaction between intracortical inhibition and facilitation in human motor cortex. J Physiol (Lond) 496:873-881.

Ziemann U, Ilic TV, Pauli C, Meintzschel F, Ruge D (2004) Learning modifies subsequent induction of long-term potentiation-like and long-term depression-like plasticity in human motor cortex. J Neurosci 24:16661672. 